\title{
Sordera súbita: A la luz de la evidencia
}

\author{
Sudden deafness: In light of the evidence
}

\author{
Consuelo Urrejola C'1, Cristian Papuzinski A², Ricardo Wegman V².
}

\begin{abstract}
RESUMEN
La sordera súbita es una condición de hipoacusia neurosensorial aguda usualmente unilateral, de instalación en un período menor o igual a 72 horas, que en la mayoría de los casos es idiopática; sin embargo, causas infecciosas, autoinmunes y vasculares, se han propuesto como los principales mecanismos involucrados. La sordera súbita es considerada una urgencia otorrinolaringológica, sin embargo el tratamiento de ésta continúa siendo un tema controvertido.
\end{abstract}

Palabras clave: Sordera súbita, hipoacusia, corticoterapia.

\begin{abstract}
Sudden deafness is usually a condition of acute unilateral sensorineural hearing loss, installation less than or equal to 72 hours period, which in most cases are idiopathic; however, infectious, autoimmune and vascular causes, have been proposed as key mechanisms involved. Sudden deafness is considered an ENT urgency, however this treatment remains controversial.
\end{abstract}

Key words: Sudden deafness, hearing loss, corticotherapy.

\section{EPIDEMIOLOGÍA}

La incidencia anual de la sordera súbita se estima en 2 a 20 casos por $100.000^{1-3}$, afectando más frecuentemente a personas entre 43 y 53 años, sin diferencias por $\operatorname{sex}^{4}$. Con un porcentaje de recuperación espontánea entre $32 \%$ y $65 \%{ }^{5}$.
No existen datos actualizados de la incidencia nacional, siendo una patología ampliamente subdiagnosticada3,6. Tampoco existe evidencia categórica de los factores de riesgo implicados ${ }^{7}$.

La sordera súbita es un concepto amplio en el marco de una hipoacusia aguda neurosensorial. La definición más aceptada corresponde a la

1 Interna de Medicina, Universidad de Valparaíso.

2 Médico Otorrinolaringología, Hospital Van Buren, Universidad de Valparaíso.

Recibido el 18 de febrero de 2015. Aceptado el 1 de marzo de 2015. 
planteada por el National Institutes of Health (NIH) que especifica los siguientes criterios: hipoacusia idiopática, de al menos $30 \mathrm{~dB}$, en 3 o más frecuencias medidas, en un período de 72 horas 8 . Esta definición ha sido adoptada por las principales guías clínicas ${ }^{2}$ y es la más utilizada en ensayos clínicos; no obstante, se debe considerar entre sus limitantes, que asume audición normal previo a la instalación de los síntomas.

\section{ETIOLOGÍA}

La etiología de la sordera súbita en más del $70 \%$ de los casos es desconocida ${ }^{9-11}$. Existen diversas teorías respecto a los mecanismos involucrados en base a estudios de series de casos, que incluyen inflamación viral de la cóclea ${ }^{12-14}$, infecciones bacterianas, enfermedades autoinmunes ${ }^{14,16}$, eventos microvasculare ${ }^{17-18}$, sustancias ototóxicas, neoplasias, trauma y otras causas variadas ${ }^{14-18}$; sin embargo, no existe evidencia concluyente ni consenso respecto de éste punto.

\section{CLÍNICA}

La hipoacusia es generalmente de inicio súbito o de rápida instalación, siendo en la mayoría de los casos unilateral, reportándose compromiso bilateral en menos de $5 \%{ }^{1,17}$. La pérdida auditiva puede comprometer las frecuencias altas, bajas 0 ser global. Los síntomas asociados más frecuentes son la sensación de plenitud aural y el tinnitus ${ }^{2,5,19}$. Síntomas como vértigo y mareo han sido descritos entre $20 \%$ y $60 \%{ }^{20}$, y en menor frecuencia otalgia o parestesias ${ }^{1}$.

\section{DIAGNÓSTICO}

La sordera súbita muchas veces es subdiagnosticada o presenta un retraso en el diagnóstico, esto debido a que es indolora, su sintomatología es inespecífica y sus síntomas tienden a ser atribuidos porel pacientey/o médicosacausascotidianas, como infecciones respiratorias, alergias, impactación de cerumen, entre otras. El cuadro de sordera súbita se sospecha en un paciente con clínica concordante y ausencia de causas identificables por la historia y examen físico otorrinolaringológico normal, por lo tanto el enfrentamiento se basa en el descarte de otras etiologías². En la anamnesis se debe indagar antecedentes de trauma, otalgia, supuración, fiebre, focalidad neurológica, cefalea, diplopía, dolor ocular; las cuales no son manifestaciones atribuibles a la sordera súbita ${ }^{21-23}$. La hipoacusia fluctuante sugiere otra causa, como enfermedad de Ménière, síndrome de hiperviscosidad, enfermedades autoinmunes u otras menos frecuentes ${ }^{12,23}$. Luego se debe realizar un examen físico detallado, considerando: otoscopía para descartar causas locales, como otitis externa, otitis media, perforación timpánica o colesteatoma; examen neurológico, centrado en la evaluación de pares craneanos y pruebas cerebelosas para distinguir el infarto del territorio de la arteria cerebelosa anteroinferior ${ }^{2}$. Las pruebas de Rinne y Weber permiten definir si la hipoacusia es de conducción o neurosensorial.

Finalmente se requerirá del apoyo de exámenes complementarios. La audiometría es requisito en todos estos pacientes, ya que además de confirmar la hipoacusia neurosensorial, permite establecer severidad, pronóstico y una herramienta de seguimiento ${ }^{2}$. Adicionalmente el estudio con resonancia magnética contrastada permite descartar otras causas que no pueden ser identificadas en las evaluaciones previas, enfermedad de Ménière, esclerosis múltiple, insuficiencias vasculares, fístulas

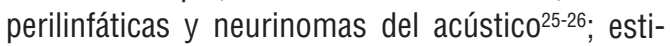
mándose que entre 1\%-4\% de los neurinomas del acústico debutan con una sordera súbita ${ }^{21,22,34}$. Si no es posible realizar una resonancia magnética, la tomografía computarizada puede suplir su función, teniendo en cuenta su menor capacidad diagnóstica en este contexto ${ }^{25,26}$. También en el diagnóstico diferencial se debe considerar a la primera crisis de un hidrops endolinfático, lo cual puede ser corroborado con una electrococleografía positiva ${ }^{27}$.

\section{TRATAMIENTO}

No existe evidencia categórica que pruebe la efectividad de las diferentes líneas de tratamiento sugeridas en el manejo de la sordera súbita. Sin embargo, guías internacionales avalan el uso de glucocorticoides sistémicos como primera 
línea de tratamiento ${ }^{2-4}$. La administración oral se recomienda para el manejo inicial, mientras que la administración intratimpánica clásicamente se reserva como terapia de rescate 0 ante la presencia de contraindicaciones para el uso de glucocorticoides sistémicos en altas dosis ${ }^{28}$.

A pesar de ser la primera línea de tratamiento, la efectividad de los corticoides orales permanece incierta ${ }^{2,28}$. Clásicamente existen dos estudios clínicos aleatorizados respecto a este tema, el primero de ellos respalda la efectividad en pacientes con hipoacusia leve y moderada ${ }^{5}$, mientras el segundo no demostró diferencias versus el grupo control ${ }^{29}$. Otros estudios no aleatorizados han mostrado utilidad con la terapia de corticoides sistémicos en la recuperación de la audición, tanto a corto como a largo plazo ${ }^{30,31}$. Revisiones sistemáticas, con metaanálisis, que incluyen estos estudios, concluyen que aún no existe evidencia que apoye el uso de corticoides por sobre el placebo $0^{30-34}$. Además se plantea dentro de las limitantes: la variabilidad en el diseño, tamaño de las poblaciones y la validez metodológica de algunos de estos ensayos ${ }^{32-34}$. Sin desmedro de lo anterior, las guías clínicas sugieren tratamiento con prednisona $1 \mathrm{mg} / \mathrm{kg} / \mathrm{día}$ por 10-14 días², aunque en algunos casos se sugiere extender la terapia por 10 días más si la respuesta es parcial ${ }^{35}$. Adicionalmente se ha observado que el tratamiento diario presenta igual efectividad que el tratamiento en pulsos, sin presentar mayores efectos adversos ${ }^{36-38}$.

El tratamiento con glucocorticoides intratimpánicos ha demostrado utilidad como tratamiento en aquellos pacientes con respuesta escasa tras la administración sistémica y en caso de contraindicación a la corticoterapia sistémica ${ }^{28,32,39-64}$. Sin embargo, los resultados varían según los criterios utilizados para evaluar la recuperación ${ }^{44}$, y no existen estudios que evalúen la significancia clínica de la mejoría descrita en las pruebas audiométricas. Además, el beneficio de los corticoides intratimpánicos se ha observado en pacientes con sordera moderada a severa, excluyendo los casos de sordera profunda ${ }^{37,38}$.

Recientemente estudios han demostrado que los corticoides intratimpánicos como tratamiento inicial no presentan diferencias al tratamiento convencional, teniendo el beneficio de evitar las reacciones adversas del tratamiento sistémico $0^{51,53-59}$.
Las limitaciones de estos estudios son la exclusión de casos de sordera profunda y el pequeño número de pacientes reclutados.

En relación al uso de terapia conjunta de corticoides sistémicos e intratimpánicos existe evidencia a favor que sugiere mejores resultados en cuanto a pruebas auditivas, especialmente en casos de sordera severa ${ }^{54,60-62}$, mientras que otros estudios han demostrado que no existen diferencias al compararla con la terapia sistémica exclu$\operatorname{siva}^{48,49,55,57}$. Por tanto este punto continúa siendo controvertido.

La terapia antiviral ha sido propuesta como un complemento al tratamiento convencional con glucocorticoides, debido al discutido rol de agentes virales en la patogenia de la sordera súbita. Sin embargo, la evidencia actual no ha demostrado beneficio al administrarse concomitantemente con corticoides $^{65-68}$.

Existen otras alternativas de tratamiento que se han planteado considerando las posibles etiologías del cuadro. El carbógeno inhalado y lipo-prostaglandina E1 en un estudio prospectivo demostraron que la terapia triple (esteroide, carbógeno y LPE1) es superior a la convencional y a la biterapia (esteroide-carbógeno) ${ }^{69}$. Por otro lado, un estudio retrospectivo postula la asociación esteroide-carbógeno por sobre la esteroide-LPE1 ${ }^{70}$. Sin embargo, no existe claridad respecto a la efectividad de estas intervenciones debido a la diferencia de diseño de los estudios y el escaso número de pacientes reclutados ${ }^{69}$.

La combinación de fibrinógeno y lipoproteína de baja densidad (LDL) demostró efectividad planteándose como alternativa al tratamiento convencional. Además la administración de éstos asociados al tratamiento con corticoides, demostró superioridad en relación al grupo control ${ }^{69}$.

La utilización de oxígeno hiperbárico como terapia ha sido debatida. Una revisión sistemática reciente demostró mejoría en la audiometría, pero se desconoce su relevancia clínica. Al mismo tiempo, se constató que la técnica no ofrece utilidad en pacientes en fase crónica ${ }^{71}$.

El uso de magnesio y antioxidantes vía oral (vitamina $E$ y $C$ ) han demostrado beneficio en estudios pequeños ${ }^{72}$.

En relación al uso de vasodilatadores y sustancias vasoactivas, los ensayos clínicos que los 
consideran $^{2}$, presentan un alto riesgo de sesgo debido a su metodología y pequeño tamaño de la muestra, no pudiendo llegar a una conclusión respecto de su eficacia.

\section{PRONÓSTICO Y SEGUIMIENTO}

Se describe que la sordera súbita tiene un buen pronóstico, especialmente en pacientes con afectación exclusiva de altas o de bajas frecuencias. Por el contrario, aquellos pacientes con hipoacusia profunda transversal a todas las frecuencias presentan un pronóstico reservado ${ }^{73}$. Dentro de los predictores clínicos destacan la edad avanzada y la presencia de vértigo como predictores de mal pronóstico ${ }^{73}$. La presencia de tinnitus, en cambio, y una curva ascendente a la audiometría se asocian a mayores porcentajes de recuperación ${ }^{74-75}$.

Se describe que al menos dos tercios de los pacientes presentan algún grado de mejoría, siendo a menudo parcial ${ }^{1,76}$, la cual se hace evidente en la mitad de los casos dentro de los primeros días ${ }^{76}$.

En cuanto al seguimiento, se sugiere realizar control con audiometría a los 6 meses, postulándose la utilidad de controles tempranos, sólo en pacientes que han sido tratados de forma precoz².

\section{BIBLIOGRAFÍA}

1. Fetterman Bl, Luxford WM, Saunders JE. Sudden bilateral sensorineural hearing loss. The Laryngoscope 1996; 106(11): 1347-50. Epub 1996/11/01.

2. Stachler RJ, Chandrasekhar SS, Archer SM, Rosenfeld RM, Schwartz SR, Barrs DM, et al. Clinical practice guideline: sudden hearing loss. Otolaryngology--head and neck surgery: Official Journal of American Academy of Otolaryngology-Head and Neck Surgery. 2012; 146(3 Suppl): S1-35. Epub 2012/03/14.

3. Artal R, Hernández R, Royo J, Vallés V. Sordera súbita: Estudio retrospectivo sobre 40 casos ingresados en nuestro hospital. Rev Otorrinolaringol Cir Cabeza Cuello 2012; 72 : 39-48.

4. RАUCH SD. Clinical practice. Idiopathic sudden sensorineural hearing loss. The New England
Journal of Medicine 2008; 359(8): 833-40. Epub 2008/08/22.

5. Wilson WR, ByL FM, LaIRD N. The efficacy of steroids in the treatment of idiopathic sudden hearing loss. A double-blind clinical study. Archives of Otolaryngology 1980; 106(12): 7726. Epub 1980/12/01.

6. Simmons FB. Sudden idiopathic sensorineural hearing loss: some observations. The Laryngoscope 1973; 83(8): 1221-7. Epub 1973/08/01.

7. Nakashima T, Tanabe T, Yanagita N, Wakal K, Ohno Y. Risk factors for sudden deafness: a case-control study. Auris, nasus, larynx 1997; 24(3): 265-70. Epub 1997/07/01.

8. Health. NIo. Sudden Deafness. Bethesda, MD. National Institutes of Health 2000; NIH publication 00-4757.

9. Chau JK, Lin JR, Atashband S, Irvine RA, Westerberg BD. Systematic review of the evidence for the etiology of adult sudden sensorineural hearing loss. The Laryngoscope 2010; 120(5): 1011-21. Epub 2010/04/28.

10. Correa A, Pacheco A. Hipoacusia Súbita Idiopática. Rev Otorrinolaringol Cir Cabeza Cuello 2000; Vol 60: $14-2$.

11. Wilson WR, VeltrI RW, Laird N, SprinkLe PM. Viral and epidemiologic studies of idiopathic sudden hearing loss. Otolaryngology--head and neck surgery: Official Journal of American Academy of Otolaryngology-Head and Neck Surgery 1983; 91(6): 653-8. Epub 1983/12/01.

12. Fowler KB, McCollister FP, Dahle AJ, Boppana $S$, BRITT WJ, PASS RF. Progressive and fluctuating sensorineural hearing loss in children with asymptomatic congenital cytomegalovirus infection. The Journal of Pediatrics 1997; 130(4): 624-30. Epub 1997/04/01.

13. López M, Lanas A, Albertz N, Piemonte P, Vergara I. Etiología viral de la hipoacusia sensorioneural súbita: ¿Mito o realidad? Rev Otorrinolaringol Cir Cabeza Cuello 2011; 71: 215-22.

14. Toubi E, Ben-David J, Kessel A, Halas K, Sabo E, Luntz M. Immune-mediated disorders associated with idiopathic sudden sensorineural hearing loss. The Annals of Otology, Rhinology, and Laryngology 2004; 113(6): 445-9. Epub 2004/07/01.

15. Baek MJ, Park HM, Johnson JM, Altuntas CZ, Jane-WIT D, JaInI R, ET AL. Increased frequencies 
of cochlin-specific $T$ cells in patients with autoimmune sensorineural hearing loss. Journal of Immunology 2006; 177(6): 4203-10. Epub 2006/09/05.

16. Capaccio P, Ottaviani F, Cuccarini V, Bottero A, Schindler A, Cesana BM, et al. Genetic and acquired prothrombotic risk factors and sudden hearing loss. The Laryngoscope 2007; 117(3): 547-51. Epub 2007/03/06.

17. Fusconi M, Chistolini A, Angelosanto N, Pignoloni P, Tombolini M, De Virgillo A, et AL. Role of genetic and acquired prothrombotic risk factors in genesis of sudden sensorineural hearing loss. Audiology \& Neuro-Otology 2011; 16(3): 18590. Epub 2010/08/28.

18. Kellerhals B. Acoustic trauma and cochlear microcirculation. An experimental and clinical study on pathogenesis and treatment of inner ear lesions after acute noise exposure. Advances in Oto-Rhino-Laryngology 1972; 18: 91-168. Epub 1972/01/01.

19. Rambold H, Boenki J, Stritzke G, Wisst F, Neppert B, HeLmChen C. Differential vestibular dysfunction in sudden unilateral hearing loss. Neurology 2005; 64(1): 148-51. Epub 2005/01/12.

20. IsaACSON JE, Vora NM. Differential diagnosis and treatment of hearing loss. American Family Physician 2003; 68(6): 1125-32. Epub 2003/10/04.

21. Sauvaget E, Kici S, Kania R, Hermán P, Tran BA, Huy P. Sudden sensorlneural Hearing loss as a revealing symptom of vestibular Schwannoma. Acta Otolaryngol 2005; 125: 592-5.

22. Stott C, Albertz N, Aedo C. Neurinoma del acústico (schwanoma vestibular): Revisión y actualización de la literatura. Rev Otorrinolaringol Cir Cabeza Cuello 2008; 68: 301-8.

23. Reiss M, Reiss G. [Differential diagnosis of unilateral hearingloss]. Praxis 2000; 89(6): 2417. Epub 2000/04/28. Zur Differential diagnose der ein seitigen Schwerhorigkeit.

24. PeÑA A. Las sentencias más comunes en otorrinolaringología. Rev Otorrinolaringol Cir Cabeza Cuello 2014; 74: 167-80.

25. Weber PC, Zbar RI, Gantz BJ. Appropriateness of magnetic resonance imaging in sudden sensorineural hearing loss. Otolaryngology-head and neck surgery: Official Journal of American Academy of Otolaryngology-Head and Neck Surgery. 1997; 116(2): 153-6. Epub 1997/02/01.

26. Daniels RL, Swallow C, Shelton C, Davidson HC, Krejci CS, Harnsberger HR. Causes of unilateral sensorineural hearing loss screened by highresolution fast spin echo magnetic resonance imaging: review of 1,070 consecutive cases. The American Journal of Otology 2000; 21(2): 173-80. Epub 2000/03/25.

27. Gamboa D, PÁez. Evaluación del vértigo y/o trastornos del equilibrio en pacientes con presión endolinfática. Revista Areté 2013; 13(1): 3248.

28. González R, Caro J. Corticoides intratimpánicos: una revisión sistemática. Rev Otorrinolaringol Cir Cabeza Cuello 2007; 67: 178-85.

29. Cinamon U, Bendet E, Kronenberg J. Steroids, carbogen or placebo for sudden hearing loss: a prospective double-blind study. European archives of oto-rhino-laryngology: Official Journal of the European Federation of Oto-RhinoLaryngological Societies 2001; 258(9): 477-80. Epub 2002/01/05.

30. Chen CY, Halpin C, Rauch SD. Oral steroid treatment of sudden sensorineural hearing loss: a ten year retrospective analysis. Otology \& Neurotology: Official Publication of the American Otological Society, American Neurotology Society [and] European Academy of Otology and Neurotology 2003; 24(5): 728-33. Epub 2003/09/23.

31. Filipo R, Attanasio G, Cagnoni L, Masci E, Russo FY, CARTOCCI G, ET AL. Long-term results of intratympanic prednisolone injection in patients with idiopathic sudden sensorineural hearing loss. Acta Oto-Laryngologica 2013; 133(9): 900-4. Epub 2013/05/23.

32. Conlin AE, Parnes LS. Treatment of sudden sensorineural hearing loss: II. A Meta-analysis. Archives of Otolaryngology--Head \& Neck Surgery 2007; 133(6): 582-6. Epub 2007/06/20.

33. Conlin AE, Parnes LS. Treatment of sudden sensorineural hearing loss: I. A systematic review. Archives of Otolaryngology--Head \& Neck Surgery 2007; 133(6): 573-81. Epub 2007/06/20.

34. Crane Ra, Camilon M, Nguyen S, Meyer ta. Steroids for treatment of sudden sensorineural hearing loss: A meta-analysis of randomized controlled trials. The Laryngoscope 2014. Epub 2014/07/22. 
35. Wei BP, Stathopoulos D, O'Leary S. Steroids for idiopathic sudden sensorineural hearing loss. The Cochrane database of systematic reviews 2013; 7: CD003998. Epub 2013/07/03.

36. Labus J, Breil J, Stutzer H, Michel 0. Metaanalysis for the effect of medical therapy vs. placebo on recovery of idiopathic sudden hearing loss. The Laryngoscope 2010; 120(9): 1863-71. Epub 2010/08/31.

37. ARTS HA. Sensorineural hearing loss: Evaluation and management in adults. Cummings $\mathrm{CW}$, Flint PW, Harker LA, Haughey BH, Richardson MA, Robbins KT, et al Cummings Otolaryngology Head and Neck Surgery $4^{\text {th }}$ ed Philadelphia: Elsevier Mosby. 2005; 3550-5.

38. Westerlaken B0, de Kleine E, van der Laan B, Albers F. The treatment of idiopathic sudden sensorineural hearing loss using pulse therapy: a prospective, randomized, double-blind clinical trial. The Laryngoscope 2007; 117(4): 684-90. Epub 2007/04/07.

39. SpeAR SA, Schwartz SR. Intratympanic steroids for sudden sensorineural hearing loss: a systematic review. Otolaryngology--head and neck surgery: Official Journal of American Academy of Otolaryngology-Head and Neck Surgery 2011; 145(4): 534-43. Epub 2011/08/30.

40. Filipo R, Attanasio G, Russo Fy, Viccaro M, Mancini P, CoveluI E. Intratympanic steroid therapy in moderate sudden hearing loss: a randomized, triple-blind, placebo-controlled trial. The Laryngoscope 2013; 123(3): 774-8. Epub 2013/02/05.

41. Garavello W, Galluzzi F, Gaini RM, Zanetti D. Intratympanic steroid treatment for sudden deafness: a meta-analysis of randomized controlled trials. Otology \& Neurotology: official publication of the American Otological Society, American Neurotology Society [and] European Academy of Otology and Neurotology 2012; 33(5): 724-9. Epub 2012/06/16.

42. Wu HP, Chou YF, Yu SH, Wang CP, Hsu CJ, Chen PR. Intratympanic steroid injections as a salvage treatment for sudden sensorineural hearing loss: a randomized, double-blind, placebo-controlled study. Otology \& Neurotology: official publication of the American Otological Society, American Neurotology Society [and] European Academy of Otology and Neurotology 2011; 32(5): 774-9. Epub 2011/06/08.
43. Chou YF, Chen PR, Kuo IJ, Yu SH, Wen YH, Wu HP. Comparison of intermittent intratympanic steroid injection and near-continual transtympanic steroid perfusion as salvage treatments for sudden sensorineural hearing loss. The Laryngoscope 2013; 123(9): 2264-9. Epub 2013/06/28.

44. Choung YH, Park K, Shin YR, Cho MJ. Intratympanic dexamethasone injection for refractory sudden sensorineural hearing loss. The Laryngoscope 2006; 116(5): 747-52. Epub 2006/05/03.

45. Cvorovic L, Jovanovic MB, Milutinovic Z, Arsovic N, DJeRIC D. Randomized prospective trial of hyperbaric oxygen therapy and intratympanic steroid injection as salvage treatment of sudden sensorineural hearing loss. Otology \& Neurotology: official publication of the American Otological Society, American Neurotology Society [and] European Academy of Otology and Neurotology 2013; 34(6): 1021-6. Epub 2013/07/04.

46. Haynes DS, O'Malley M, Cohen S, Watford K, LABADIE RF. Intratympanic dexamethasone for sudden sensorineural hearing loss after failure of systemic therapy. The Laryngoscope 2007; 117(1): 3-15. Epub 2007/01/05.

47. Hunchaisri N, Chantapant S, SRinangyam N. Intratympanic dexamethasone for refractory sudden sensorineural hearing loss. Journal of the Medical Association of Thailand = Chotmaihetthangphaet 2010; 93(12): 1406-14. Epub 2011/02/25.

48. Lee JB, Chol SJ, Park K, Park HY, Choo OS, Choung $\mathrm{YH}$. The efficiency of intratympanic dexamethasone injection as a sequential treatment after initial systemic steroid therapy for sudden sensorineural hearing loss. European archives of oto-rhinolaryngology: Official Journal of the European Federation of Oto-Rhino-Laryngological Societies 2011; 268(6): 833-9. Epub 2011/01/12.

49. LI L, REN J, YIN T, LIU W. Intratympanic dexamethasone perfusion versus injection for treatment of refractory sudden sensorineural hearing loss. European archives of oto-rhinolaryngology: Official Journal of the European Federation of Oto-Rhino-Laryngological Societies 2013; 270(3): 861-7. Epub 2012/06/07.

50. Park MK, Lee CK, Park KH, Lee JD, Lee CG, Lee BD. Simultaneous versus subsequent intratympa- 
nic dexamethasone for idiopathic sudden sensorineural hearing loss. Otolaryngology-head and neck surgery: Official Journal of American Academy of Otolaryngology-Head and Neck Surgery 2011; 145(6): 1016-21. Epub 2011/08/06.

51. Seggas I, Koltsidopoulos P, Bibas A, Tzonou A, Sismanis A. Intratympanic steroid therapy for sudden hearing loss: a review of the literature. Otology \& Neurotology: official publication of the American Otological Society, American Neurotology Society [and] European Academy of Otology and Neurotology 2011; 32(1): 29-35. Epub 2010/12/31.

52. Wang YW, ReN JH, Lu YD, YIN TF, XIEDH. Evaluation of intratympanic dexamethasone for treatment of refractory sudden sensorineural hearing loss. Journal of Zhejiang University Science B 2012; 13(3): 203-8. Epub 2012/03/01.

53. Han CS, Park JR, Boo SH, Jo JM, Park KW, Lee WY, ET AL. Clinical efficacy of initial intratympanic steroid treatment on sudden sensorineural hearing loss with diabetes. Otolaryngology-head and neck surgery: Official Journal of American Academy of Otolaryngology-Head and Neck Surgery 2009; 141(5): 572-8. Epub 2009/10/29.

54. BATTISTA RA. Intratympanic dexamethasone for profound idiopathic sudden sensorineural hearing loss. Otolaryngology--head and neck surgery: official Journal of American Academy of Otolaryngology-Head and Neck Surgery 2005; 132(6): 902-5. Epub 2005/06/10.

55. Filipo R, attanasio G, Russo Fy, Cartocci G, Musacchio A, De Carlo A, et al. Oral versus Short-Term Intratympanic Prednisolone Therapy for Idiopathic Sudden Hearing Loss. Audiology \& Neuro-Otology 2014; 19(4): 225-33. Epub 2014/07/06.

56. Koltsidopoulos P, Bibas A, Sismanis A, Tzonou A, SEgGAS I. Intratympanic and systemic steroids for sudden hearing loss. Otology \& Neurotology: official publication of the American Otological Society, American Neurotology Society [and] European Academy of Otology and Neurotology 2013; 34(4): 771-6. Epub 2013/05/09.

57. Lim HJ, Kim YT, Cho SJ, Lee JB, Park HY, Park K, ET AL. Efficacy of 3 different steroid treatments for sudden sensorineural hearing loss: a prospective, randomized trial. Otolaryngology--head and neck surgery: Official Journal of American Academy of Otolaryngology-Head and Neck Surgery 2013; 148(1): 121-7. Epub 2012/10/19.

58. Rauch SD, Halpin CF, Antonelli PJ, Babu S, Carey JP, Gantz BJ, et al. Oral vs intratympanic corticosteroid therapy for idiopathic sudden sensorineural hearing loss: a randomized trial. JAMA: the Journal of the American Medical Association 2011; 305(20): 2071-9. Epub 2011/05/26.

59. Bae SC, Noh HI, Jun BC, Jeon EJ, Seo JH, Park Sy, ET AL. Efficacy of intratympanic steroid therapy for idiopathic sudden sensorineural hearing loss: comparison with systemic steroid therapy and combined therapy. Acta Oto-Laryngologica 2013; 133(5): 428-33. Epub 2013/01/30.

60. Dispenza F, Amodio E, De Stefano A, Gallina S, Marchese D, Mathur N, et al. Treatment of sudden sensorineural hearing loss with transtympanic injection of steroids as single therapy: a randomized clinical study. European archives of oto-rhino-laryngology: Official Journal of the European Federation of Oto-RhinoLaryngological Societies 2011; 268(9): 1273-8. Epub 2011/02/18.

61. Labatut T, Daza MJ, Alonso A. Intratympanic steroids as primary initial treatment of idiopathic sudden sensorineural hearing loss. The Hospital Universitario Ramon y Cajal experience and review of the literature. European archives of oto-rhino-laryngology: Official Journal of the European Federation of Oto-RhinoLaryngological Societies 2013; 270(11): 282332. Epub 2012/12/21.

62. Battaglia A, Burchette R, Cueva R. Combination therapy (intratympanic dexamethasone + highdose prednisone taper) for the treatment of idiopathic sudden sensorineural hearing loss. Otology \& Neurotology: official publication of the American Otological Society, American Neurotology Society [and] European Academy of Otology and Neurotology 2008; 29(4): 453-60. Epub 2008/04/11.

63. Gundogan 0, Pinar E, Imre A, Ozturkcan S, COKMEZ 0, Yigiter AC. Therapeutic efficacy of the combination of intratympanic methylprednisolone and oral steroid for idiopathic sudden deafness. Otolaryngology--head and neck surgery: Official 
Journal of American Academy of OtolaryngologyHead and Neck Surgery 2013; 149(5): 753-8. Epub 2013/08/21.

64. SuzUKI H, Hashida K, NguYen KH, НоhCHI N, КатоH A, KoIzUmı H, ET AL. Efficacy of intratympanic steroid administration on idiopathic sudden sensorineural hearing loss in comparison with hyperbaric oxygen therapy. The Laryngoscope 2012; 122(5): 1154-7. Epub 2012/03/27.

65. Awad Z, Huins C, Pothier DD. Antivirals for idiopathic sudden sensorineural hearing loss.

The Cochrane database of systematic reviews 2012; 8: CD006987. Epub 2012/08/17.

66. Uri N, Doweck I, Cohen-Kerem R, Greenberg E. Acyclovir in the treatment of idiopathic sudden sensorineural hearing loss. Otolaryngology-head and neck surgery: Official Journal of American Academy of Otolaryngology-Head and Neck Surgery. 2003; 128(4): 544-9. Epub 2003/04/23.

67. Westerlaken BO, Stokroos RJ, Dhooge IJ, Wit HP, ALBERS FW. Treatment of idiopathic sudden sensorineural hearing loss with antiviral therapy: a prospective, randomized, double-blind clinical trial. The Annals of Otology, Rhinology, and Laryngology 2003; 112(11): 993-1000. Epub 2003/12/05.

68. Stokroos RJ, Albers FW, Tenvergert EM. Antiviral treatment of idiopathic sudden sensorineural hearing loss: a prospective, randomized, doubleblind clinical trial. Acta Oto-Laryngologica 1998; 118(4): 488-95. Epub 1998/09/03.

69. KIM MG, Jung YG, Eun YG. Effect of steroid, carbogen inhalation, and lipoprostaglandin E1 combination therapy for sudden sensorineural hearing loss. American Journal of Otolaryngology 2011; 32(2): 91-5. Epub 2009/12/22.

70. Lee HJ, Park CY, Lee JH, Yang HS, Kim JH, Ban MJ, ET AL. Therapeutic effects of carbogen inhalation and lipo-prostaglandin E1 in sudden hearing loss. Yonsei Medical Journal 2012; 53(5): 9991004. Epub 2012/08/08.

71. Agarwal L, Pothier DD. Vasodilators and vasoactive substances for idiopathic sudden sensorineural hearing loss. The Cochrane database of systematic reviews 2009(4): CD003422. Epub 2009/10/13.

72. Nageris BI, Ulanovski D, Attias J. Magnesium treatment for sudden hearing loss. The Annals of Otology, Rhinology, and Laryngology 2004; 113(8): 672-5. Epub 2004/08/28.

73. Wen YH, Chen PR, Wu HP. Prognostic factors of profound idiopathic sudden sensorineural hearing loss. European archives of oto-rhinolaryngology: Official Journal of the European Federation of Oto-Rhino-Laryngological Societies 2014; 271(6): 1423-9. Epub 2013/06/19.

74. Ben-David J, Luntz M, Magamsa I, Fradis M, Sabo E, Podoshin L. Tinnitus as a prognostic sign in idiopathic sudden sensorineural hearing loss. The International Tinnitus Journal 2001; 7(1): 62-4. Epub 2004/02/18.

75. Ben-David J, Luntz M, Podoshin L, Sabo E, Fradis M. Vertigo as a prognostic sign in sudden sensorineural hearing loss. The International Tinnitus Journal 2002; 8(2): 127-8. Epub 2004/02/07.

76. BYL FM, JR. Sudden hearing loss: eight years' experience and suggested prognostic table. The Laryngoscope 1984; 94(5 Pt 1): 647-61. Epub 1984/05/01. 